\title{
First report of vampyrellid predator-prey dynamics in a marine system
}

\author{
Catharina Alves-de-Souza $\mathbb{D}^{1,2} \cdot$ Tatiane S. Benevides $^{2} \cdot$ Mariângela Menezes $^{2} \cdot$ Christian Jeanthon $^{3} \cdot$ Laure Guillou $^{3}$
}

Received: 26 September 2018 / Revised: 21 November 2018 / Accepted: 23 November 2018 / Published online: 6 December 2018

(c) International Society for Microbial Ecology 2018

\begin{abstract}
We report for the first time the in situ dynamics of a vampyrellid in a marine system. A high sampling frequency (twice-weekly) was applied in a tropical eutrophic lagoon (Rio de Janeiro, Brazil) for 5 years (2012-2016). The vampyrellid Hyalodiscus sp. specifically fed on the diatom Chaetoceros minimus during a short time window ( 3 months), although the prey was intermittently detected as the dominant phytoplanktonic species over a longer period ( 1 year). A classic Lotka-Volterra predator-prey dynamic was observed between the two partners, with a significant modification of the shortterm oscillations of the prey. Specific abiotic preferences (i.e., relatively low temperature, intermediate salinity, and stratified conditions) associated with prey availability seemed to define this narrow temporal window of occurrence. Our results suggest that vampyrellids can be ecologically relevant in marine pelagic systems, with their impact on planktonic dynamics strongly depending on complex interactions between both biotic and abiotic factors.
\end{abstract}

Vampyrellids (Vampyrellida, Rhizaria) are a relatively easily recognizable group of predatory amoebae feeding on protists and small metazoans in aquatic and terrestrial systems [1]. They display an impressive diversity of feeding strategies varying from prey engulfment to protoplast feeding, the latter characterized by the perforation of the prey cell wall and protoplast extraction [2]. As all formally described vampyrellid species so far have been recorded in freshwater or soil ecosystems, the group was initially thought to be confined to non-marine habitats [2-4]. However, recent molecular surveys revealed a high diversity of these organisms in marine microbial assemblages [1]. Although the understanding of

Supplementary information The online version of this article (https:// doi.org/10.1038/s41396-018-0329-0) contains supplementary material, which is available to authorized users.

Catharina Alves-de-Souza

cathsouza@gmail.com

1 Algal Resources Collection, MARBIONC, Center for Marine Sciences, University of North Carolina Wilmington, 5600 Marvin K. Moss Lane, Wilmington, NC 28409, USA

2 Laboratório de Ficologia, Departamento de Botânica, Museu Nacional/Universidade Federal do Rio de Janeiro, Quinta da Boa Vista S/N, São Cristóvão, Rio de Janeiro, RJ 20940-040, Brazil

3 CNRS \& Sorbonne Université, Station Biologique de Roscoff, Place Georges Teissier, CS90074, 29688 Roscoff, France vampyrellid phylogeny and life history has improved substantially in recent years [3,5-7], little is known about their abundance and impact on prey populations. Consumption of plankton prey has been observed mainly through feeding experiments [6] or algal mass cultures [7]. The putative genus Asterocaelum is the only known example of a vampyrellid preying on freshwater natural plankton assemblages, where they are frequently reported as a significant source of mortality for diatoms and cyanobacteria [8-11].

Here we present the first record of the high-frequency predator-prey dynamics for a vampyrellid in a marine system. We followed the phytoplankton dynamics in Rodrigo de Freitas Lagoon (RFL), a eutrophic coastal system in Rio de Janeiro (Brazil), from January 2012 to December 2016. Samplings were performed twice-weekly at five sampling stations (Fig. S1; see Supplementary Materials for methodological details). This lagoon is dominated by small eukaryotic phytoplankton $(<20 \mu \mathrm{m})$ and high temporal variability [12]. The diatom Chaetoceros minimus was one of these dominant species highly fluctuating over the time series (Fig. 1a). Predation on C. minimus by a vampyrellid was first detected in July 2013 in all the sampling stations. Microscopic observations revealed that only $C$. minimus was consumed by Hyalodiscus sp. in both field populations and feeding experiments using cultures from other microalgal species occurring in RFL (listed in supplementary materials). Although studies on vampyrellids' prey range are still 

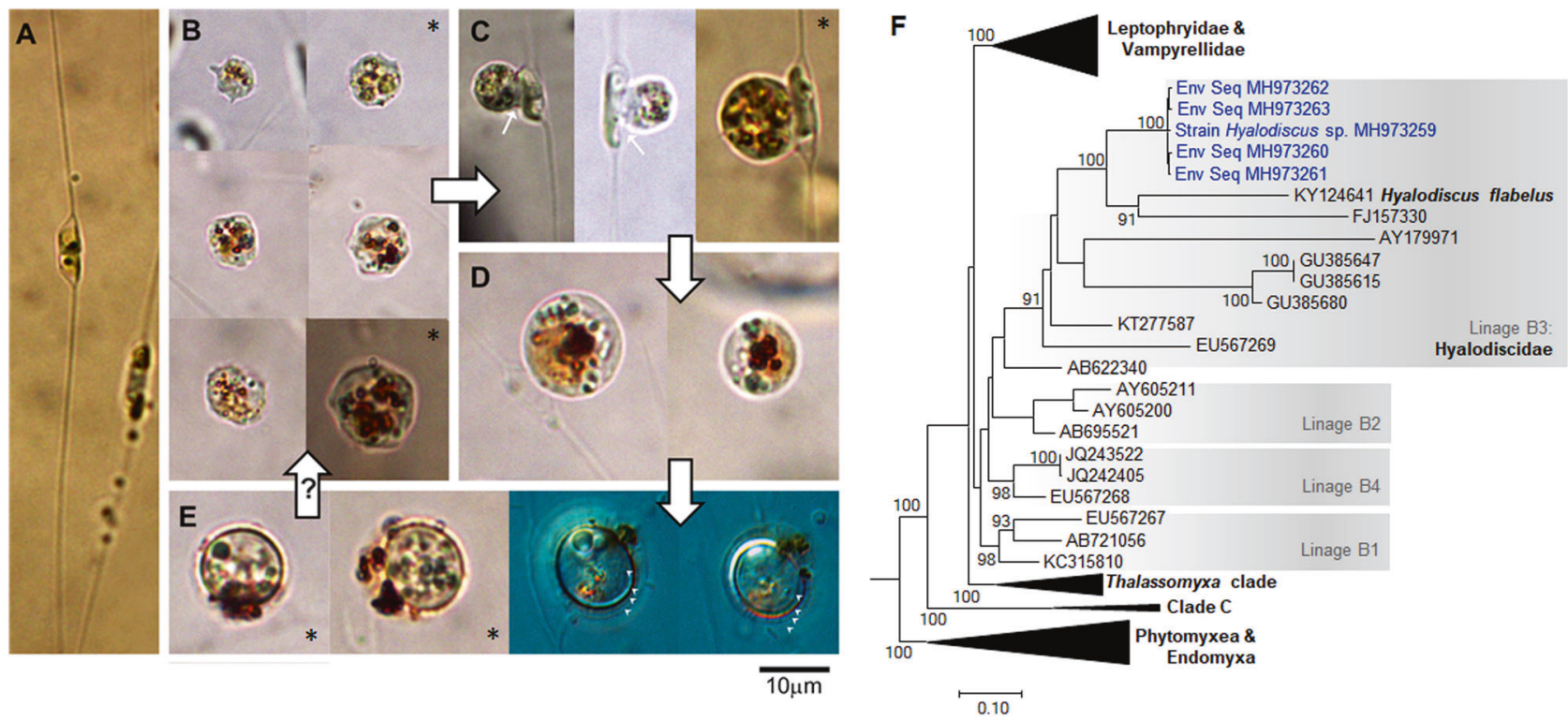

Fig. 1 a Healthy diatom Chaetoceros minimus, the only microalgae observed to be predated by Hyalodiscus sp. in mixed plankton assemblage from the Rodrigo de Freitas Lagoon (RFL) over the time series. b-e Different stages of Hyalodiscus sp.: trophozoite (amoeboid freeliving, feeding stage) (b), feeding stage attached to C. minimus cells (c), digestive cysts (d), and resting cysts (e). f Maximal likelihood phylogeny (SSU rDNA) of vampyrellids showing the inclusion of Hyalodiscus sp. sequences (in blue) into Hyalodiscidae. Only bootstrap values $>70 \%$ are shown (expanded tree is given in Fig. S2; see Supplementary Material for details on the analysis). Small white arrows = feeding peduncle; large black arrows $=$ order of transition between the different Hyalodiscus sp. stages as observed in cultures from RFL (transition from resting cysts to the free-living stage is depicted with a question mark as it was not observed in cultures); arrow heads indicate the four layers of resting cyst envelops. Asterisks = individuals recorded from Lugols' samples during quantification (others originated from cultures) insipient, both broad ranges of preys, including fungi, algae, and nematodes [13, 14], and trophic specialization [6] were reported. Protoplast-feeding vampyrellids have been observed so far feeding on large-sized algae [3, 5, 6, 15]. To the best of our knowledge, this is the first report of a vampyrellid with such feeding strategy preying on a nanosized planktonic microalgae.

A culture of the vampyrellid (now lost) was established using a $C$. minimus strain previously isolated from RFL as prey. Typical vampyrellid life stages were recognized from wild and cultured populations (Fig. 1b-e). Both trophozoites (amoeboid free-living, feeding stages) and digestive cysts having orange cytoplasmic colouration are typical for vampyrellids (e.g., refs. [3, 5]). The vampyrellid was identified as Hyalodiscus sp. based on the sequencing of the small subunit ribosomal DNA (SSU rDNA) of the obtained culture. SSU rRNA gene sequences affiliated to Hyalodiscus sp. accounted for $20-50 \%$ of the clone libraries (data not shown) when the highest abundances of the vampyrellid occurred. Maximum likelihood analysis (Fig. 1f, Fig. S2) indicated that SSU rRNA gene sequences of the cultured and environmental vampyrellids grouped together within the Hyalodiscus flabelus clade (100\% bootstrap) and fell into the linage B3 [1], which was recently suggested as encompassing the family Hyalodiscidae [5]. Sequences belonging to this clade have been reported worldwide in sediments of coastal systems [1].
We observed a strong interannual environmental heterogeneity in RFL, mainly related to salinity and BruntVäisälä frequency ( $N_{\mathrm{BV}}$; an estimate of stratification) (Fig. 2a). Dynamics of the main phytoplankton groups also reflected such variability (Fig. 2b). Of special notice was the long period of intermittent $C$. minimus predominance (June 2013 to August 2014) (Fig. 2c) related to high $\mathrm{Si}(\mathrm{OH})_{4}$ (Figs. S3, S4). Hyalodiscus sp. was observed only between July and August 2013 (Fig. 2d). The high sampling frequency allowed the detection of classic Lotka-Volterra dynamics between $C$. minimus and Hyalodiscus sp. (Fig. 2e), with a 6-day time lag $\left(t_{-6}\right)$ between the abundance peaks of the prey and its predator (cross-correlation $t_{-6}=$ $0.7 ; p>0.01$; Fig. S5). No natural enemies (i.e., grazers or parasites) other than Hyalodiscus sp. showed such a coupled dynamic with $C$. minimus during its period of predominance (Alves-de-Souza, data not shown). Wavelet coherence analysis demonstrated a significant time-delayed negative interaction between prey and predator (Fig. S6), whereas multiple polynomial regressions identified the lagged $\left(t_{-6}\right)$ Hyalodiscus sp. abundance as the main factor affecting the abundance of its diatom prey during the period of their co-occurrence $\left(R^{2}=0.40 ; p<0.001\right.$; Table S1).

Hyalodiscus sp. showed a narrow niche breadth when compared to its prey (Fig. 2f), which was related to an apparent preference of the vampyrellid for temperatures 

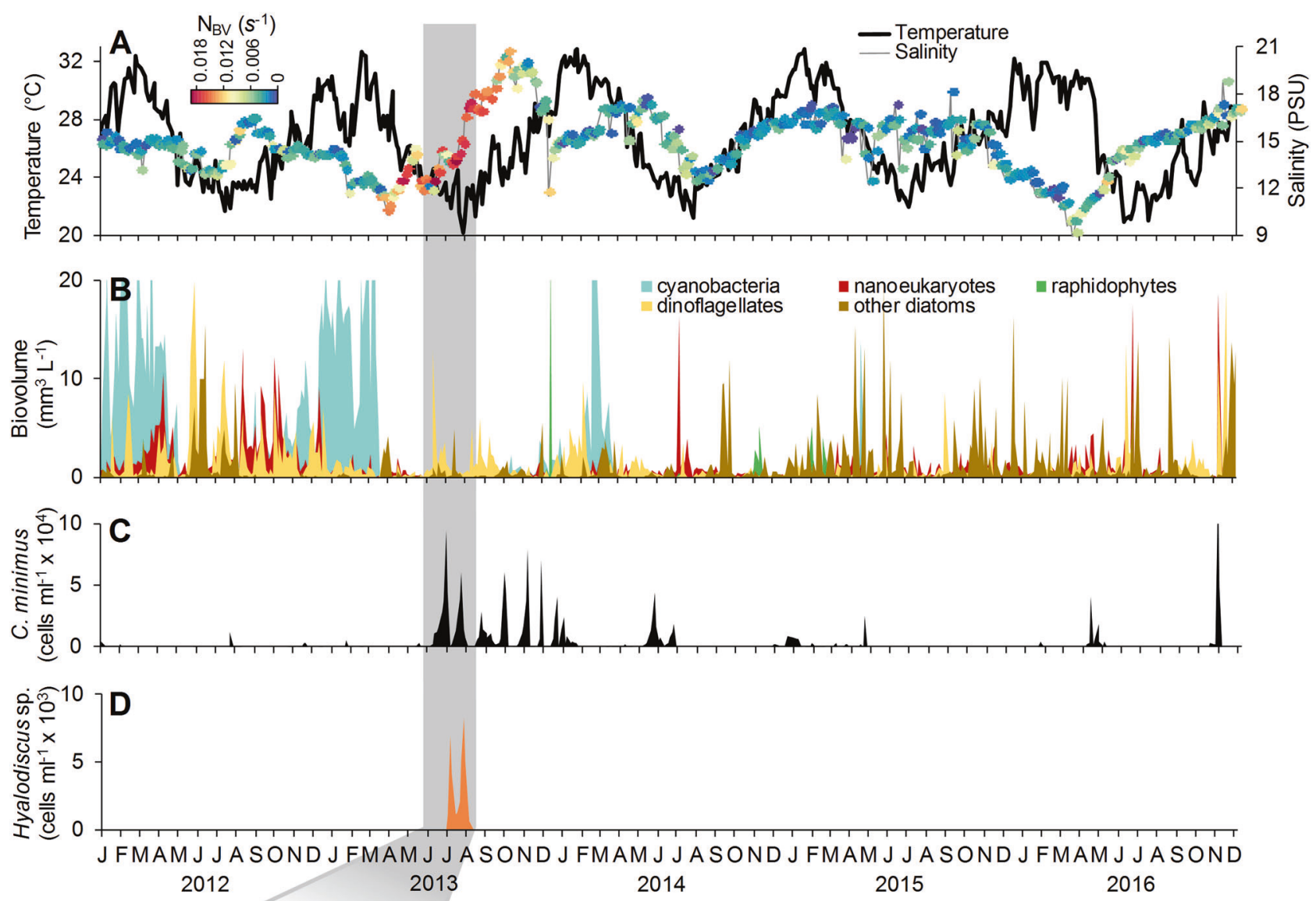

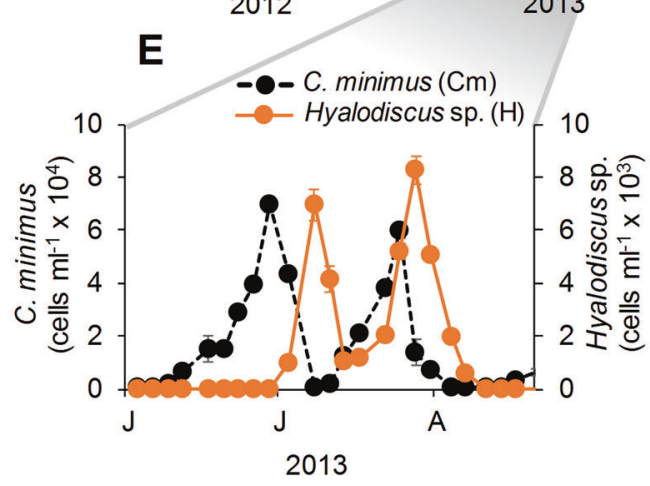

Fig. 2 a, b Interannual and seasonal variability of average values (for the five sampling stations) of water temperature $\left({ }^{\circ} \mathrm{C}\right)$, salinity (PSU), Brunt-Väisälä frequency $\left(N_{\mathrm{BV}}\right)\left(\mathrm{s}^{-1}\right)$ as an estimate of water stratification and phytoplankton biovolume $\left(\mathrm{mm}^{3} \mathrm{~L}^{-1}\right)$. c, d Average abundance (cells $\mathrm{ml}^{-1}$ ) of Chaetoceros minimus (c) and Hyalodiscus sp. (d). e Detail showing the time-lagged interaction between $C$. minimus and Hyalodiscucs sp. (grey area in a-d). f Outlying mean index (OMI) analysis showing the relative importance of environmental variables

$<23{ }^{\circ} \mathrm{C}$, intermediate salinities (12-16 PSU) and stratified conditions $\left(N_{\mathrm{BV}}>0.01 \mathrm{~s}^{-1}\right)$ (Fig. $\left.2 \mathrm{~g}\right)$. This precise combination of abiotic parameters was observed only during Hyalodiscus sp. occurrence. Polynomial regressions based on environmental variables and time lagged values $\left(t_{-6}\right)$ of C. minimus abundance indicated that Hyalosdiscus sp. abundance was mostly affected by the interaction between temperature, $N_{\mathrm{BV}}$ and lagged $\left(t_{-6}\right) C$. minimus abundance $\left(R^{2}=0.95 ; p>0.01\right.$; Table $\left.\mathrm{S} 2\right)$. A slightly different pattern was obtained for the period of $C$. minimus predominance, with interaction between temperature and lagged $\left(t_{-6}\right)$ $C$. minimus abundance, explaining most variability in Hyalodiscus sp. abundance (partial $R^{2}=0.75 ; p>0.01$ ). These results indicated that $N_{\mathrm{BV}}$ was mostly important at the 
interannual scale and partly explained the restricted occurrence of Hyalodiscus sp. in 2013. However, the importance of this variable decreased when a shorter time period was considered because the water column was mostly stratified during the period of predominance of $C$. minimus. During this restricted time window, the increase in temperature may explain why Hyalodiscus disappeared even when the water column was still stratified and the prey present. Shifts in the relative importance of the environmental variables according to temporal scale have been also previously reported for other microbial communities [16, 17], including phytoplankton assemblages of RFL [12].

The restricted temporal occurrence of Hyalodiscus sp. in the RFL was in agreement with an extensive study on vampyrellids' diversity in marine systems where only $13 \%$ of the vampyrellid sequences were recorded from pelagic samples, with the major relative abundance (87\%) for this group detected in sediments [1]. While information on microbial benthic assemblages is not yet available for RFL, further studies should access the relevance of resting cysts in the sediments for the Hyalodiscus sp. predator-prey dynamics in this system. The conjunction of specific abiotic conditions (i.e., relatively low temperature, intermediate salinity and stratified waters) associated with specific prey availability seemed to determine the time window for Hyalodiscus sp. pelagic presence in RFL. Considering the worldwide distribution of vampyrellids within the Hyalodiscidae clade [1] and the cosmopolitan nature of $C$. minimus [18], our results provide valuable insights into the population dynamics of vampyrellids and the factors behind their elusive occurrence in plankton assemblages. They also suggest that this group can indeed be ecologically relevant in marine plankton systems, with their relative importance to the planktonic dynamics depending strongly on complex interactions between both biotic and abiotic factors.

Acknowledgements We are grateful to the Secretary of Environment of the Municipality of Rio de Janeiro for allowing us access to the data used in this work. We also thank Wendy Strangman for the English review of the manuscript. This work was funded by the Brazilian National Council for Scientific and Technological Development (CNPq) (14/2014 446687/2014-6, PDJ/CNPq503443/2012-3 to CA-d$\mathrm{S})$ and the International Research Network "Diversity, Evolution and Biotechnology of Marine Algae" (GDRI No. 0803 to LG and CA-d-S).

\section{Compliance with ethical standards}

Conflict of interest The authors declare that they have no conflict of interest.

\section{References}

1. Berney C, Romac S, Mahé F, Santini S, Siano R, Bass D. Vampires in the oceans: predatory cercozoan amoebae in marine habitats. ISME J. 2013;7:2387-99.

2. Hülsmann N. Lateromyxa gallica n. g., n. sp.(Vampyrellidae): a filopodial amoeboid protist with a novel life cycle and conspicuous ultrastructural characters. J Eukaryot Microbiol. 1993;40:141-9.

3. Hess S, Sausen N, Melkonian M. Shedding light on vampires: the phylogeny of vampyrellid amoebae revisited. PLoS ONE. 2012;7: e31165.

4. Anderson T, Patrick Z. Mycophagous amoeboid organisms from soil that perforate spores of Thielaviopsis basicola and Cochliobolus sativus. Phytopathology. 1978;68:1618-26.

5. Hess S. Description of Hyalodiscus flabellus sp. nov.(Vampyrellida, Rhizaria) and identification of its bacterial endosymbiont, "Candidatus Megaira polyxenophila" (Rickettsiales, Alphaproteobacteria). Protist. 2017a;168:109-33.

6. Hess S. Hunting for agile prey: trophic specialisation in leptophryid amoebae (Vampyrellida, Rhizaria) revealed by two novel predators of planktonic algae. FEMS Microbiol Ecol. 2017. https://doi.org/10.1093/femsec/fix104.

7. Gong Y, Patterson DJ, Li Y, Hu Z, Sommerfeld M, Chen Y, et al. Vernalophrys algivore gen. nov., sp. nov.(Rhizaria: Cercozoa: Vampyrellida), a new algal predator isolated from outdoor mass culture of Scenedesmus dimorphus. Appl Environ Microbiol. 2015;81:3900.

8. Van Wichelen J, Muylaert K, Van Der Gucht K, Vyverman W. Observations on little studied protists (chytrids and an amoeba), affecting phytoplankton populations in the upper reaches of the Schelde Estuary (Belgium). Belgian J Bot. 2006;139:153-66.

9. Bailey-Watts A, Lund J. Observations on a diatom bloom in Loch Leven, Scotland. Biol J Linn Soc. 1973;5:235-53.

10. Canter HM. A new primitive protozoan devouring centric diatoms in the plankton. Zool J Linn Soc. 1973;52:63-83.

11. Cook WL, Ahearn DG. Natural control of Anabaena blooms by the amoeba Asterocaelum anabaenophilum sp. nov. In: Parker BC, Roane KM, editors. Algae and fungi. Biogeography, systematics, and ecology. Charlottesville, VA: University Press of Charlottesville; 1976. p. 71-80.

12. Alves-de-Souza C, Benevides TS, Santos JB, Von Dassow P, Guillou L, Menezes M. Does environmental heterogeneity explain temporal $\beta$ diversity of small eukaryotic phytoplankton? Example from a tropical eutrophic coastal lagoon. J Plankton Res. 2017; 39:698-714.

13. Old K, Darbyshire J. Soil fungi as food for giant amoebae. Soil Biol Biochem. 1978;10:93-100.

14. Pakzad U. Untersuchungen einer mykophagen Vampiramöbe, $\mathrm{PhD}$ dissertation, University of Giessen, Germany. 2003.

15. Cienkowski L. Ueber einige rhizopoden und verwandte organismen. Arch Mikrosk Anat. 1876;12:15-50.

16. Reynolds C. Temporal scales of variability in pelagic environments and the response of phytoplankton. Freshw Biol. 1990;23:25-53.

17. Hatosy SM, Martiny JB, Sachdeva R, Steele J, Fuhrman JA, Martiny AC. Beta diversity of marine bacteria depends on temporal scale. Ecology. 2013;94:1898-904.

18. Tomas CR. Identifying marine phytoplankton. San Diego, CA: Academic Press; 1997. 